University of Wollongong

Research Online

Faculty of Engineering and Information

Faculty of Engineering and Information

Sciences - Papers: Part A

Sciences

$1-1-2014$

\title{
A novel type of one-dimensional organic selenium-containing fiber with superior performance for lithium-selenium and sodium-selenium batteries
}

Hongqiang Wang

University of Wollongong, hw571@uowmail.edu.au

Sha Li

University of Wollongong, sl739@uowmail.edu.au

Zhixin Chen

University of Wollongong, zchen@uow.edu.au

Hua-Kun Liu

University of Wollongong, hua@uow.edu.au

Zaiping Guo

University of Wollongong, zguo@uow.edu.au

Follow this and additional works at: https://ro.uow.edu.au/eispapers

Part of the Engineering Commons, and the Science and Technology Studies Commons

Research Online is the open access institutional repository for the University of Wollongong. For further information contact the UOW Library: research-pubs@uow.edu.au 


\title{
A novel type of one-dimensional organic selenium-containing fiber with superior performance for lithium-selenium and sodium-selenium batteries
}

\author{
Abstract \\ A novel type of organic selenide fiber composed of carbonized polyacrylonitrile/selenium (CPAN/Se) has \\ been synthesized by heating polyacrylonitrile-selenium (PAN-Se) fibers via the electrospinning technique \\ at $600^{\circ} \mathrm{C}$. The Se molecules are confined by $\mathrm{N}$-containing carbon ring structures in the form of energy- \\ storing selenium side chains in the carbonized PAN matrix. This unique stable chemical structure with a \\ conductive carbon skeleton connected to the selenium side chains and excellent mechanical stability can \\ allow CPAN/Se composite cathodes to be charged and discharged in a low-cost carbonate-based \\ electrolyte with excellent long cycle stability and quite good rate performance. The superior \\ electrochemical performance of CPAN/Se electrodes is demonstrated in both lithium-ion and sodium-ion \\ batteries, where they have delivered a high capacity of nearly $600 \mathrm{~mA} \mathrm{~h} \mathrm{g-1} \mathrm{for} 500$ cycles in lithium- \\ selenium (Li-Se) batteries and $410 \mathrm{~mA} \mathrm{~h} \mathrm{g-1} \mathrm{for} 300$ cycles in sodium-selenium ( $\mathrm{Na}-\mathrm{Se}$ ) batteries at $0.3 \mathrm{C}$, \\ respectively.

\section{Keywords} \\ lithium, sodium, performance, superior, type, fiber, novel, containing, selenium, organic, dimensional, one, \\ batteries \\ Disciplines \\ Engineering | Science and Technology Studies

\section{Publication Details} \\ Wang, H., Li, S., Chen, Z., Liu, H. Kun. \& Guo, Z. (2014). A novel type of one-dimensional organic selenium- \\ containing fiber with superior performance for lithium-selenium and sodium-selenium batteries. RSC \\ Advances: an international journal to further the chemical sciences, 4 (106), 61673-61678.
}




\title{
A Novel Type of One-Dimensional Organic Selenium-Containing Fibers with Superior Performance for Lithium-Selenium and Sodium-Selenium Batteries
}

\author{
Hongqiang Wang, ${ }^{a}$ Sha Li, ${ }^{a}$ Zhixin Chen, ${ }^{a}$ Hua Kun Liu ${ }^{a}$ and Zaiping Guo ${ }^{* a, b, c}$ \\ 5 Received (in $X X X, X X X) X$ th $X X X X X X X X X 20 X X$, Accepted $X$ th $X X X X X X X X X 20 X X$ \\ DOI: $10.1039 / b 000000 x$
}

\begin{abstract}
A novel type of organic selenide fibers composed of carbonized polyacrylonitrile/selenium (CPAN/Se) have been synthesized by heating polyacrylonitrile-selenium (PAN-Se) fibers via the electrospinning technique at $600{ }^{\circ} \mathrm{C}$. The Se molecules are confined by $\mathrm{N}$-containing carbon ring 10 structures in the form of energy-storing selenium side chains in the carbonized PAN matrix. This unique stable chemical structure with a conductive carbon skeleton connected to the selenium side chains and excellent mechanical stability can allow CPAN/Se composite cathodes to be charged and discharged in a low-cost carbonate-based electrolyte with excellent long cycle stability and quite good rate performance. The superior electrochemical performance of CPAN/Se electrodes is demonstrated in both lithium-ion 15 and sodium-ion batteries, where it has delivered a high capacity of nearly $600 \mathrm{mAh} \mathrm{g}^{-1}$ for 500 cycles in lithium-selenium (Li-Se) batteries and $410 \mathrm{mAh} \mathrm{g}^{-1}$ for 300 cycles in sodium-selenium (Na-Se) batteries at $0.3 \mathrm{C}$, respectively.
\end{abstract}

\section{Introduction}

The rechargeable lithium-sulfur (Li-S) battery has attracted 20 enormous attention in the past several years due to its high energy density and ultra-high theoretical specific capacity (2567 Wh kg-1 and $1675 \mathrm{mAh} \mathrm{g}^{-1}$, respectively). ${ }^{1-3}$ Selenium, as a congener of sulfur, possesses similar chemical behavior and could react with lithium to form selenides. The theoretical gravimetric capacity of 25 selenium electrode $\left(675 \mathrm{mAh} \mathrm{g}^{-1}\right)$ is lower than that of sulfur, however, the theoretical volumetric capacity of selenium (3253 $\mathrm{mAh} \mathrm{cm}^{-3}$ ) is comparable to that of sulfur (3467 $\left.\mathrm{mAh} \mathrm{cm}^{-3}\right){ }^{4-7}$ More importantly, the electrical conductivity of selenium is much higher than that of sulfur. ${ }^{5}$ These features of Se make it an 30 attractive alternative candidate for cathode material in lithium-ion batteries as well as sodium-ion batteries. Due to the similarity to sulfur, however, the selenium cathodes also suffer from the dissolution issue of high-order polyselenides, leading to the low utilization of selenium, low coulombic efficiency of the selenium 35 cathode, and fast capacity fading.

At present, study on selenium cathode is at a very early stage. Several attemps have been applied to enhance the electrochemical properties of selenium-based cathodes. Amine's group ${ }^{5}$ firstly conducted work on Se/CNT composite as a cathode material, and 40 the Se/CNT composite electrode could deliver a capacity of 300 $\mathrm{mAh} \mathrm{g}^{-1}$ after 100 cycles for rechargeable lithium battery. Guo et al. ${ }^{6}$ prepared Se/CMK-3 composite with a reversible capacity of $600 \mathrm{mAh} \mathrm{g}^{-1}$ after 50 cycles by using a simple thermal-treatment method. Wang et al. $^{7}$ synthesized nano-Se ${ }_{8}$-carbon composite 45 containing $30 \%$ selenium and the obtained $\mathrm{Se}_{8} / \mathrm{C}$ composite electrodes demonstrated excellent electrochemical performance and could remain capacity of $480 \mathrm{mAh} \mathrm{g}^{-1}$ in Li-Se batteries for 1000 cycles, and $340 \mathrm{mAh} \mathrm{g}^{-1}$ in Na-Se batteries for 380 cycles. To date, all the research on the selenium cathode for energy 50 storage has been focused on "physical" methods to confine selenium in a porous matrix to prevent soluble polyselenides from dissolving in the organic electrolyte. ${ }^{5-10}$ It is clear that trapping selenium in the porous matrix by such a physical method is an effective approach to solve the dissolution problem. On the 55 other hand, using a "chemical" method involving functional groups and the unique chain structure of the polymer maybe a more efficient strategy to confine the selenium compared to the physical method because it can yield excellent stability and activity. Herein, we report a novel type of organic selenium60 containing fiber material, in which the Se is trapped in energystoring selenium side chains in an organic carbon skeleton matrix. This novel fibrous material was prepared by heating polyacrylonitrile-Se (PAN-Se) fibers via the electrospinning technique under vacuum, in which trigonal Se is converted into 65 chain-like $\mathrm{Se}_{\mathrm{x}}$ molecular groups that are bonded to the carbonized polymer during the calcination process. The conductive carbon skeleton can effectively confine the selenium by forming a stable structure connected to the selenium side chains. Given the high electrochemical activity of the selenium side chains linked to the 70 organic carbon and the strong interaction between them, this novel selenium cathode shows excellent stability during lengthy electrochemical cycling for both lithium-ion and sodium-ion batteries.

\section{Experimental}




\subsection{Preparation of carbonized polyacrylonitrile/selenium (CPAN/Se) fibers}

The carbonized polyacrylonitrile/selenium composite (CPAN/Se) fibers were synthesized through the electrospinning technique. In 5 a typical synthetic procedure, $1 \mathrm{~g}$ PAN was dissolved in $10 \mathrm{~g}$ dimethylformamide (DMF) and stirred for $24 \mathrm{~h}$ at $70{ }^{\circ} \mathrm{C}$ to yield a clear solution. Next, $0.8 \mathrm{~g}$ selenium powder was added into the solution. Finally, a homogeneous suspension was obtained after 4 $\mathrm{h}$ strong mechanical stirring. During electrospinning process, the 10 solution was loaded into syringe with a metallic needle (inner diameter $0.5 \mathrm{~mm}$ ) under applied voltage of $16.2 \mathrm{kV}$. The distance from needle tip to stainless steel mesh collector was about $11 \mathrm{~cm}$. Then, the collected primary film was dried at $100{ }^{\circ} \mathrm{C}$ for $24 \mathrm{~h}$. The obtained products were heat-treated at $600{ }^{\circ} \mathrm{C}$ for $5 \mathrm{~h}$ in 15 vacuum with a heating rate of $5{ }^{\circ} \mathrm{C} \mathrm{min}^{-1}$ to achieve the CPAN/Se composite. Control sample, carbonized PAN/Se powder (CPAN/Se powder), was prepared by the same process but without electrospinning technique. The PAN powders were mixed with selenium powders (5:4 by weight) and then heated at $20600{ }^{\circ} \mathrm{C}$ for $5 \mathrm{~h}$ in vacuum to obtain CPAN/Se powder.

\subsection{Materials Characterization}

The crystal structure of the samples were examined with X-ray diffraction diffractometer (XRD, MMA GBC, Australia) with Cu $\mathrm{K} \alpha$ radiation. Raman spectroscopy on an instrument (JOBIN 25 YVON HR800) equipped with a $632.8 \mathrm{~nm}$ diode laser and Fourier-transform infrared spectroscopy (FT-IR) were carried out to characterize the chemical bonds in each sample. The selenium content of composite was measured by Thermogravimetric analysis/differential scanning calorimetry (TGA/DSC) type 30 instrument (METTLER TOLEDO, Switzerland) at a heating rate of $10{ }^{\circ} \mathrm{C} \mathrm{min}^{-1}$ from room temperature to $750{ }^{\circ} \mathrm{C}$ under argon atmosphere. X-ray photoelectron spectroscopy (XPS) analysis was conducted on a VG Scientific ESCALAB 2201XL system with aluminum $\mathrm{K} \alpha \mathrm{X}$-ray radiation. JEOL JSM-7500FA Field35 emission scanning electron microscopy (FESEM) and JEOL 2011 transmission electron microscopy (TEM) were employed to investigate the morphologies of the samples.

\subsection{Electrochemical measurement}

The CPAN/Se fibers and CPAN/Se powders (75\% by weight) 40 were mixed with super-P (15\%) and PCDF (10\%) in NMP solution to form the slurry for the electrode preparation, respectively. After that, the working electrodes were prepared by coating the slurry onto aluminum foil current collector then dried at $50{ }^{\circ} \mathrm{C}$ for $24 \mathrm{~h}$. Coin-type (CR2032) cells were assembled in an 45 argon-filled glove box, with $1 \mathrm{M} \mathrm{LiPF}_{6}$ in a mixture of ethylene carbonate/diethylcarbonate (EC/DEC, 1:1, v/v) as the electrolyte for $\mathrm{Li}$-Se batteries and $1 \mathrm{M} \mathrm{NaClO}_{4}$ in a mixture of ethylene carbonate/diethyl carbonate (EC/DEC, 1:1, v/v) as the electrolyte for Na-Se batteries. Cells with CPAN electrodes were also 50 prepared using the same procedure. The coin cells were galvanostatically charged-discharged from 0.8 to $3.0 \mathrm{~V}$ for Li-Se batteries and from 0.5 to $2.5 \mathrm{~V}$ for Na-Se batteries using a cell test instrument (CT2001A, LAND, China).

\section{Results and discussions}

55 The experimental procedures are illustrated in Fig. 1. First of all, selenium powders were added into the PAN solution to form a stable suspension due to the suitable viscosity of the PAN solution, although selenium powders are insoluble in DMF (Fig. 1a). Next, one-dimensional (1D) PAN-Se fibers were prepared by 60 the electrospinning technique. Finally, the as-spun PAN-Se fibers were heated at $600{ }^{\circ} \mathrm{C}$ for $5 \mathrm{~h}$ to obtain CPAN/Se composite fibers. During the thermal treatment, the reactions of PAN with selenium are summarized as follows (Fig. 1b): When the PAN-Se fibers are heated, Se plays the role of a reducer in facilitating the 65 dehydrogenation and cyclization of PAN, resulting in a conductive main chain with generation of $\mathrm{H}_{2} \mathrm{Se}$ as a by-product. Meanwhile, the highly polar - $\mathrm{CN}$ functional groups can be cyclized in form of a stable and conductive heterocyclic compound. Subsequently, at relatively high temperature, 70 elemental selenium is subject to being cleaved into selenium free radicals and intercalated into the heterocyclic compound, which then acts as energy-storing side chains with various lengths, or to forming covalent bonds with the carbon atoms in the PANderived carbon matrix. A similar reaction has been reported in 75 sulfur-based composites. ${ }^{11-13}$ This novel type of selenium in the CPAN matrix acts as an important role in the electrochemical properties of the CPAN/Se electrodes for both Li-Se and Na-Se cells.

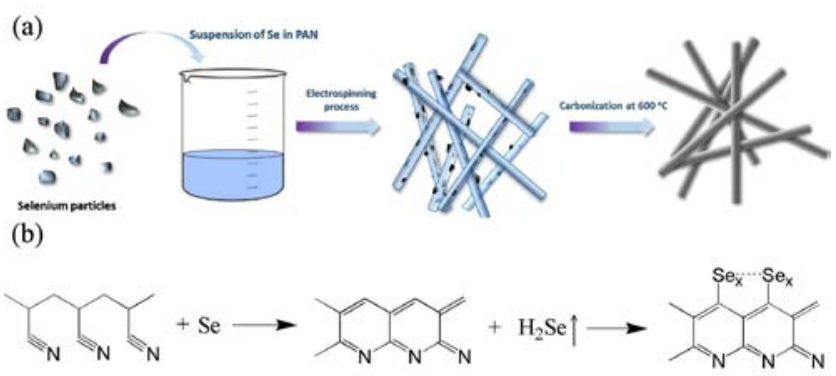

Fig. 1 (a) A schematic illustration of the experimental procedure; 80 (b) the ideal structure of CPAN/Se and its reaction process.

Fig. 2 presents SEM images of the as-spun PAN-Se fibers and

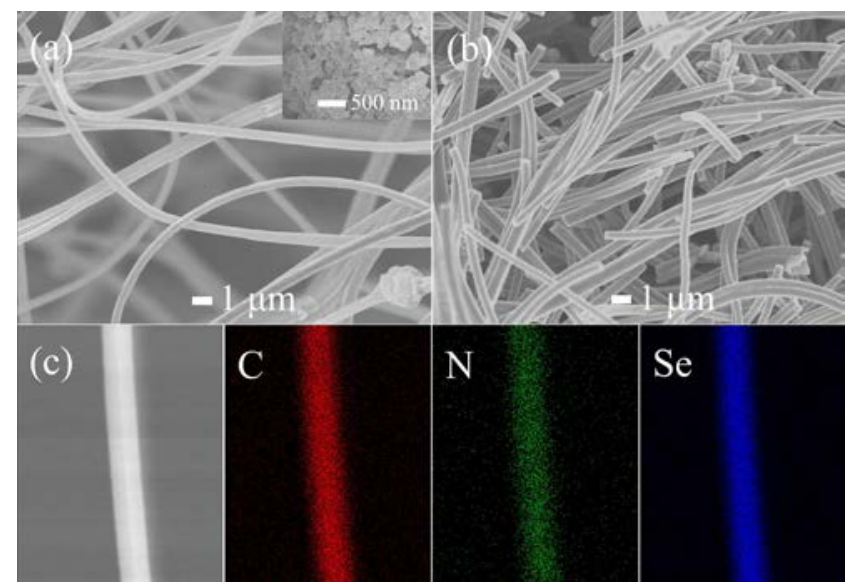

Fig. 2 SEM images of (a) as-spun PAN-Se fibers, with the inset showing an image of the selenium powders, (b) CPAN/Se fibers, and (c) a single PAN/Se fiber and the corresponding elemental 85 mapping images of carbon, nitrogen, and selenium.

CPAN/Se fibers. The as-spun PAN-Se sample has good fibrous morphology with smooth surfaces, except for a few large particles located on the surface, indicating that most of the 
selenium powders were wrapped inside of the PAN fibers during the electrospinning process. (Fig. 2a inset is an SEM image of the selenium powders, in which the particle size was mainly less than $200 \mathrm{~nm}$.) The 1D fibrous morphology was preserved during the 5 carbonization step, and no particles were left on the surfaces of the fibers, suggesting that a complete reaction between Se and PAN had occurred. The element mapping indicates that all the elements (carbon, nitrogen, and selenium) are uniformly distributed over the whole fiber.

10 Transmission electron microscopy (TEM) was employed to further investigate the microstructure of the PAN-Se and CPAN/Se fibers in Fig. 3. Before calcination, it is clear that the selenium powders are well distributed throughout the whole fiber (Fig. 3a), and high resolution TEM (HRTEM) image (Fig. 3b) 15 and selected area electron diffraction (SAED) pattern (Fig. 3c) indicate that the selenium powders are present in the form of the crystal structure in the fiber. The surface of the fiber becomes much smoother, however, and no obvious particles can be seen on the CPAN/Se fibers (Figure 3d) after the carbonization 20 process. From HRTEM image (Figure 3e) and the SAED pattern (Figure 3f) of the CPAN/Se composite, it can be concluded that the CPAN/Se composite has an amorphous structure, suggesting

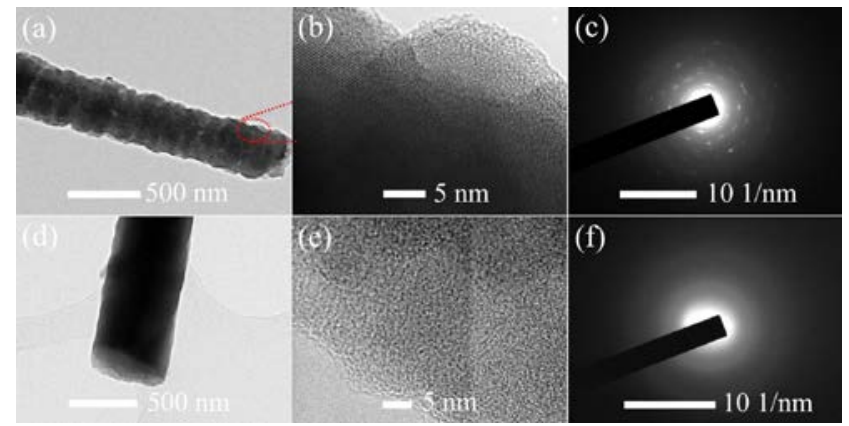

Fig. 3 TEM image (a), HRTEM image (b), and SAED pattern (c) for PAN-Se fiber; TEM image (d), HRTEM image (e), and ${ }_{25}$ SAED pattern (f) of CPAN/Se fiber.

that selenium could react with PAN during the carbonization process and exist in form of amorphous structure in the CPAN matrix.

Fig. 4a shows the XRD patterns of the elemental selenium, ${ }_{30} \mathrm{CPAN}$, and CPAN/Se. The pristine Se possesses trigonal structure, while the CPAN/Se has amorphous structure. Although there is a substantial amount of selenium (36\%, in Fig. S1 and S2) in the CPAN/Se composite, the peaks of elemental selenium could not be observed, suggesting that the embedded selenium in 35 the composite exists in the form of small molecules rather than $\mathrm{Se}_{8}$ and thus loses its characteristic of trigonal structure. Fig. $4 \mathrm{~b}$ presents the Se 3d XPS spectra for pristine Se and CPAN/Se. In the pristine Se, the Se $3 \mathrm{~d}_{5 / 2}$ and $3 \mathrm{~d}_{3 / 2}$ peaks centered at 55.8 and $56.7 \mathrm{eV}$, which could be assigned to metallic selenium. ${ }^{15}$ The Se ${ }_{40} 3 d_{5 / 2}$ and $3 d_{3 / 2}$ peaks move towards higher binding energy in the CPAN/Se, however, which means that the Se has formed bonds with other atoms in the composite to yield selenium-containing heterocyclic compounds. There is evidence in the literature that the $3 \mathrm{~d}$ peak could change when Se is combined with other atoms. 45 4,14,15

The existence of the C-Se bond is the key to judge whether Se is incorporated into the PAN-based polymer matrix. Therefore,
Raman and Fourier-transform infrared (FT-IR) spectroscopy were performed to further examine the chemical bonds in CPAN/Se. 50 Fig. 4c displays the FT-IR spectra of the CPAN and CPAN/Se. Both the CPAN and the CPAN/Se exhibit peaks at 1590, 1295, and $833 \mathrm{~cm}^{-1}$ corresponding to the $\mathrm{C}=\mathrm{C}$ bond, $\mathrm{C}=\mathrm{N}$ bond, and $\mathrm{C}$ $\mathrm{N}$ bond, respectively, ${ }^{16-19}$ suggesting the formation of a heterocyclic compound in the carbonization process. The new 55 peak at $523 \mathrm{~cm}^{-1}$ corresponds to torsion of rings in which a C-Se bond is contained, ${ }^{20}$ and the peak located at $438 \mathrm{~cm}^{-1}$ could be also attributed to the $\mathrm{C}$-Se bending vibration, ${ }^{19}$ indicating the formation of C-Se bonds after the dehydrogenation and cyclization process. In the Raman spectroscopy (Fig. 4d), the
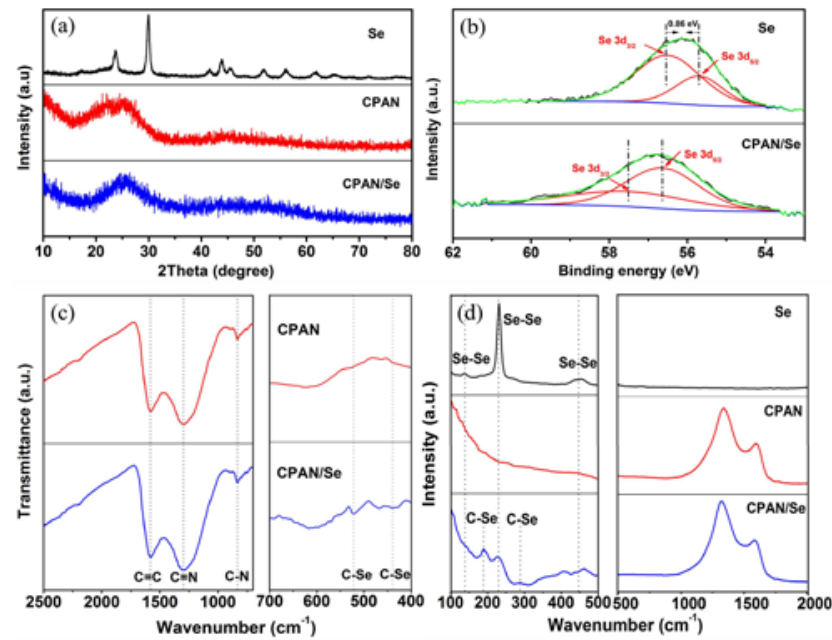

${ }_{60}$ Fig. 4 (a) XRD patterns of Se, CPAN, and CPAN/Se fibers; (b) XPS spectra of Se and CPAN/Se fibers for Se 3d; (c) FT-IR spectra of CPAN, and CPAN/Se fibers; (d) Raman spectra of Se, CPAN, and CPAN/Se fibers.

elemental selenium shows three peaks located at 138, 231, and $65458 \mathrm{~cm}^{-1}$, respectively. CPAN/Se exhibits distinctive peaks at 198 and $288 \mathrm{~cm}^{-1}$, however, indicating that the atomic configuration of CPAN/Se has changed markedly after the reaction with selenium. The peaks centered at 198 and $288 \mathrm{~cm}^{-1}$ can be attributed to the C-Se stretching vibration and the C-Se-Se group 70 vibration. $^{19}$

Cyclic voltammetry (CV) tests for CPAN/Se battaries were performed in the voltage window from $0.8 \mathrm{~V}$ to $3 \mathrm{~V}$ vs. $\mathrm{Li}^{+} / \mathrm{Li}$, and $0.5 \mathrm{~V}$ to $2.5 \mathrm{~V}$ vs. $\mathrm{Na}^{+} / \mathrm{Na}$, respectively. Typical $\mathrm{CV}$ of the CPAN/Se electrodes in Li-Se and Na-Se cells are displayed in 75 Fig. 5. There is only one pair of reversible redox peaks for Li-Se batteries in the $\mathrm{CV}$ curves, suggesting that the CPAN/Se electrode experiences a single phase transition reaction from $\mathrm{Se}_{\mathrm{x}}$ chain to insoluble $\mathrm{Li}_{2} \mathrm{Se}$ without formation of soluble intermediate phase $\mathrm{Li}_{2} \mathrm{Se}_{\mathrm{x}}(\mathrm{x} \geq 4)$ in carbonate electrolyte, which is 80 in agreement with previous reports. ${ }^{6,7}$ During the first scan, the curve shows a cathodic peak at $1.0 \mathrm{~V}$ and an anodic peak at $2.2 \mathrm{~V}$ for the Li-Se batteries. After the first scan, the cathodic peak shifts towards the positive direction, while the anodic peak remains at $2.2 \mathrm{~V}$. The interaction between organic selenium and 85 the CPAN matrix during the first lithiation may lead to more potential peak in the first cycle. It is believed that the complex bond formed between selenium and the heterocyclic compound will affect the reaction of selenium and lithium, which 

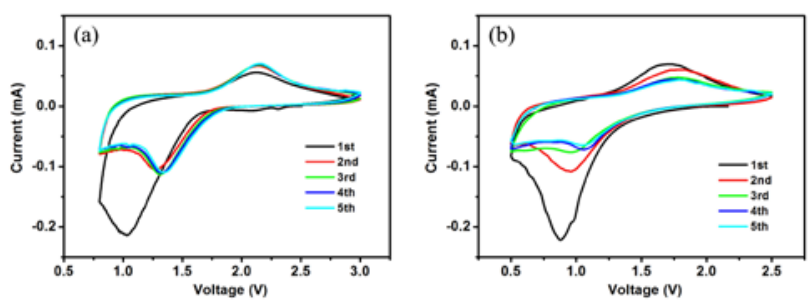

Fig. 5 Cyclic voltammograms of the CPAN/Se fiber electrodes in (a) Li-Se and (b) Na-Se batteries at a scan rate of $0.1 \mathrm{mV} / \mathrm{s}$. needs additional energy to dissociate selenium from the complex bond, leading to a more negative potential. ${ }^{12}$ The CPAN/Se cathode in Na-Se batteries exhibits similar phenomenon to that in Li-Se batteries, as well as having lower redox potentials.
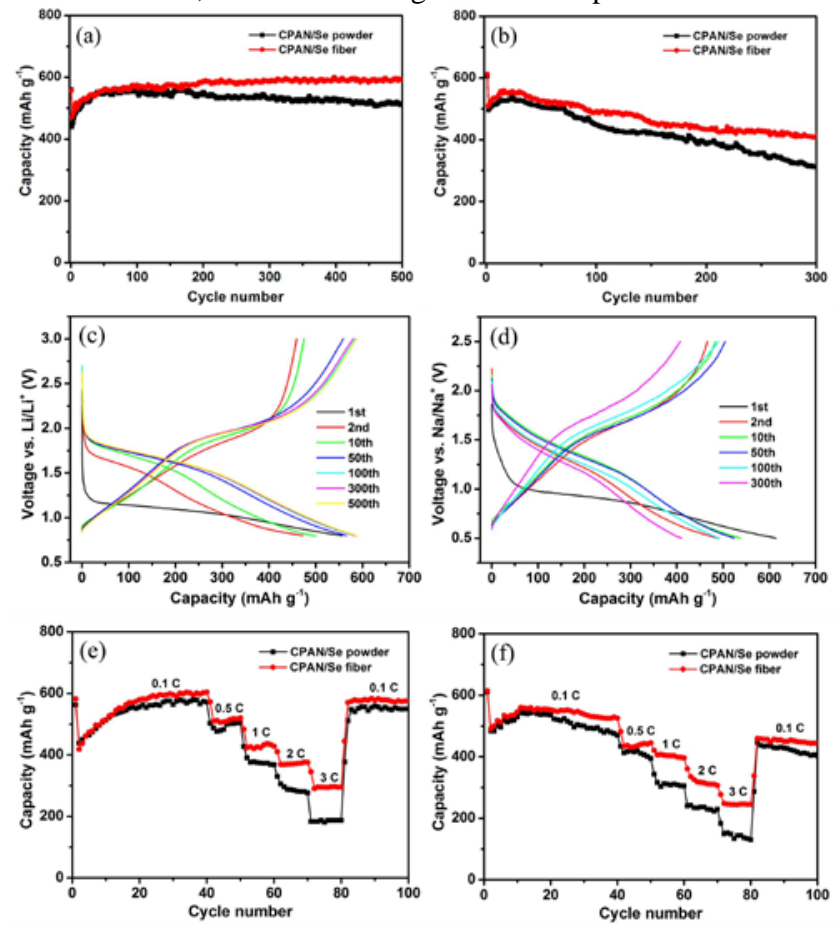

Fig. 6 Cycling performance of CPAN/Se fiber and powder 10 electrodes at $0.3 \mathrm{C}$ for (a) Li-Se and (b) Na-Se batteries; discharge/charge voltage profiles of CPAN/Se fiber electrode at selected cycles for (c) Li-Se and (d) Na-Se batteries; rate capabilities of CPAN/Se fiber and powder electrodes for (e) LiSe and (f) Na-Se batteries.

${ }_{15}$ Fig. 6a and 6b displays the cycling performance of the CPAN/Se fiber and powder cathodes in Li-Se and Na-Se batteries at $0.3 \mathrm{C}$. Excellent cycling stability in both Li-Se and Na-Se batteries can be obtained. Compared to CPAN/Se powder electrode, the CPAN/Se fiber electrode could deliver a capacity of nearly 600 $20 \mathrm{mAh} \mathrm{g}^{-1}$ without any capacity loss after 500 cycles in Li-Se batteries. Even at the rate of $1.5 \mathrm{C}$, the CPAN/Se fiber electrodes can still maintain more than $400 \mathrm{mAh} \mathrm{g}^{-1}$ for 1000 cycles (Fig. S3), indicating a superior cycling performance compared to that of pristine Se electrodes for Li-Se and Na-Se batteries (Fig. S4).

${ }_{25}$ Such superior capacity of CPAN/Se is a combined outcome from its unique chemical structure. The carbon-based skeleton matrix supports high electrical conductivity, and the PAN-derived carbon-based matrix can confine the selenium during the charge and discharge processes by forming stable energy-storing side
30 chains where selenium free radicals are intercalated into the heterocyclic compound. Furthermore, the presence of nitrogen atoms in the heterocyclic compound linked to selenium side chains could enhance the electronic conductivity, ${ }^{21,22}$ which additionally contributes to the exceptional electrochemical 35 performance. Most of all, the interaction between selenium and conductive heterocyclic compound greatly improves the cycling stability of the CPAN/Se electrodes in the low-cost carbonatebased electrolyte and provides a new strategy to develop Sebased cathodes for renewable energy storage.

40 It is worth noting that the CPAN/Se electrodes for Li-Se batteries deliver an initial discharge capacity of $560 \mathrm{mAh} \mathrm{g}^{-1}$, however, the discharge capacity drops to $460 \mathrm{mAh} \mathrm{g}^{-1}$ in the second cycle before increasing to $600 \mathrm{mAh} \mathrm{g}^{-1}$ in subsequent cycles. This interesting phenomenon is different from previous result. ${ }^{5-8}$ 45 Therefore, the electrochemical performance of CPAN electrode (Fig. S5) was tested to investigate the higher intial discharge capacity. The CPAN electrode displayed an irreversible capacity of nearly $300 \mathrm{mAh} \mathrm{g}^{-1}$ in the first discharge. A long potential plateau between $1.1 \mathrm{~V}$ and $0.8 \mathrm{~V}$ is initially present in the CPAN 50 electrode, but it disappears in the second cycle, which is in agreement with the lower plateau of CPAN/Se fiber electrode for the first discharge in Fig. 6c. This can explain its higher initial discharge capacity. In addition, the increase of capacity between the $3^{\text {rd }}$ and the $30^{\text {th }}$ cycle demonstrates a possible activation 55 process in the electrode materials. Accordingly, the increase of the capacity was accompanied by the reduced electrode impedance (Fig. S6). The fresh CPAN/Se cell shows a large charge transfer resistance. The reason is that the high degree of carbonization at higher temperature causes the active selenium to 60 be deeply enveloped within the amorphous carbon skeleton. Therefore, lithium can only access to the surface of partial active selenium. The whole active selenium could work normally again only after the activation process with longer cycles, resulting in reduced charge transfer resistance in subsequent cycles. As a 65 result, the discharge capacity is improved between the $3^{\text {rd }}$ and the $30^{\text {th }}$ cycles and the reduced impedance in the second cycle shifts the lithiation potential to a higher value as demonstrated in the CV curves in Fig. 5a. For the sodium batteries, the CPAN/Se also demonstrates good cycling performance. In this study, the ${ }_{70} \mathrm{CPAN} / \mathrm{Se}$ shows higher initial discharge capacity and a similar trend in regards to capacity to that in the Li-Se batteries, retaining $410 \mathrm{mAh} \mathrm{g}^{-1}$ after 300 cycles. Commonly, electrodes in sodiumion batteries display worse reaction kinetics and inferior electrochemical properties than that in lithium-ion batteries due to 75 the fact that the sodium ion is much bigger than lithium ion.

The rate performance of the CPAN/Se fiber and CPAN/Se powder electrodes is shown in Fig. 6e and 6f. The CPAN/Se fiber electrodes also show good rate capability in both lithium-ion and sodium-ion batteries. The control sample CPAN/Se powders were 80 synthesized through the same heat-treatment process as for CPAN/Se fibers, but based on a mixture of PAN and selenium powders. The CPAN/Se powders consist of round particles with diameter of $250 \mathrm{~nm}$ (Fig. S7 and S8). At low rates, both CPAN/Se powder and fiber electrodes show similar rate 85 performance, however, the CPAN/Se fibers exhibit superior rate performance at higher rates. In particular, the CPAN/Se fiber electrode can retain capacity of $290 \mathrm{mAh} \mathrm{g}^{-1}$ even at $3 \mathrm{C}$ and 
recover to $600 \mathrm{mAh} \mathrm{g}^{-1}$ after the rate is returned to $0.1 \mathrm{C}$, but the CPAN/Se powders just deliver $180 \mathrm{mAh} \mathrm{g}^{-1}$. The 1D fibrous nanostructure with smaller dimensions and large surface area can facilitate efficient contact between the active materials and the 5 electrolyte, and provide more active sites for electrochemical reactions. In addition, the $1 \mathrm{D}$ architecture also possesses favorable structural stability, which can help to alleviate the structural damage caused by volume expansion and to keep the integrity of the electrodes during the charge-discharge process 10 especially at high rate or after long-term cycling. All these characteristics result in such a good rate capabilities. For the NaSe batteries, the capacity of the CPAN/Se fibers is decreased from 550 to $245 \mathrm{mAh} \mathrm{g}^{-1}$ with the increase of rate from $0.1 \mathrm{C}$ to 3 C.

15 To further investigate the reason why the CPAN/Se electrode shows such a superior electrochemical performance, the microstructure of the CPAN/Se fiber electrode after 100 cycles for Li-Se batteries was examined by SEM. The fibrous structure (Fig. S9a) is still maintained, and no obvious change is observed. ${ }_{20}$ The element mapping results (Fig. S9b) show that the selenium is still homogeneously distributed throughout the whole fiber, suggesting the excellent mechanical stability of the CPAN/Se fibers. Together with its unique chemical structure, excellent electrochemical properties of CPAN/Se can be achieved.

\section{${ }_{25}$ 4. Conclusion}

In summary, a novel type of selenium fibers were simply prepared by electrospinning technique. Selenium exists in the carbonized PAN matrix in form of energy-storing selenium side chains. Such a unique chemical structure not only enhances the 30 electrical conductivity, but also can confine selenium during the charge and discharge processes, leading to superior long-term cycling stability and good rate performance. The CPAN/Se electrodes could remain a reversible capacity of $600 \mathrm{mAh} \mathrm{g}^{-1}$ for 500 cycles in Li-Se battery and $410 \mathrm{mAh} \mathrm{g}^{-1}$ for 300 cycles in Na${ }_{35}$ Se battery. In addition, this novel material exhibits superior electrochemical performance and it can be easily achieved manufacturing process, which makes our Se-based electrode highly promising for practical energy storage.

\section{Acknowledgments}

${ }_{40}$ Support of this work by the Australian Research Council (ARC) Discovery Project (DP1094261) is acknowledged. Furthermore, the authors would like to thank Dr. Tania Silver for critical reading of the manuscript and valuable remarks.

\section{Notes and references}

${ }_{45}{ }^{a}$ Institute for Superconducting \& Electronic Materials, University of Wollongong, Wollongong, Australia.E-mail: zguo@uow.edu.au

${ }^{b}$ Hubei Collaborative Innovation Center for Advanced Organic Chemical Materials, College of Chemistry and Chemical Engineering, Hubei University, Wuhan, China.

${ }_{50}{ }^{c}$ School of Mechanical, Materials \& Mechatronics Engineering, University of Wollongong, Wollongong, Australia.

$\dagger$ Electronic Supplementary Information (ESI) available: [details of any supplementary information available should be included here]. See DOI: $10.1039 / \mathrm{b} 000000 \mathrm{x} /$

551 P. G. Bruce, S. A. Freunberger, L. J. Hardwick and J. M. Tarascon, Nat. Mater., 2012, 11, 19.
2 M. K. Song, Y. G. Zhang and E. J. Cairns, Nano Lett., 2013, 13, 5891.

3 H. L. Wang, Y. Yang, Y. Y. Liang, J. T. Robinson, Y. G. Li, A. $60 \quad$ Jackson, Y. Cui and H. J. Dai, Nano Lett., 2011, 11, 2644.

4 Y. J. Cui, A. Abouimrane, J. Lu, T. Bolin, Y. Ren, W. Weng, C. J. Sun, V. A. Maroni, S. M. Heald and K. Amine, J. Am. Chem. Soc., 2013, 135, 8047.

5 A. Abouimrane, D. Dambournet, K. W. Chapman, P. J. Chupas, W. 65 Weng and K. Amine. J. Am. Chem. Soc., 2012, 134, 4505.

6 C. P. Yang, S. Xin, Y. X. Yin, H. Ye, J. Zhang and Y. G. Guo, Angew. Chem. Int. Ed., 2013, 52, 8363.

7 C. Luo, Y. H. Xu, Y. J. Zhu, Y. H. Liu, S.Y. Zheng, Y. Liu, A. Langrock and C. S. Wang, ACS Nano, 2013, 7, 8003.

708 L. L. Liu, Y. Y. Hou, X. W. Wu, S. Y. Xiao, Z. Chang, Y. Q. Yang and Y. P. Wu, Chem. Commun., 2013, 49, 11515.

9 L. L. Liu, Y. Y. Hou Y. Q. Yang, M. X. Li, X. W. Wang and Y. P. Wu, RSC Adv., 2014, 4, 9086.

10 S. F. Jiang, Z. A. Zhang, Y. Q. Lai, Y. H. Qu, X. W. Wang and J. Li, 75 J. Power Sources, 2014, 267, 394.

11 J. L.Wang, J. Yang, J. Y. Xie and N. X. Xu, Adv. Mater., 2002, 14, 963.

12 J. L.Wang, J. Yang, C. R. Wan, K. Du, J. Y. Xie and N. X. Xu, Adv. Funct. Mater., 2003, 13, 487.

8013 T. H. Hwang, D. S. Jung, J. S. Kim, B. G. Kim and J. W. Choi, Nano Lett., 2013, 13, 4532.

14 P. K. Babu, A. Lewera, J. H. Chung, R. Hunger, W. Jaegermann, N. Alonso-Vante, A. Wieckowski and E. Oldfield, J. Am. Chem. Soc., 2007, 129, 15140.

8515 J. F. Moulder, W. F. Stickle, P. E. Sobol and K. D. Bomben, Handbook of X-ray photoelectron spectroscopy, ULVAC-PHI, Inc. Chigasaki, Japan 1995.

16 X. G. Yu, J. J. Xie, Y. Li, H. J. Huang, C. Y. Lai and K. Wang, J. Power Sources, 2005, 146, 335.

9017 X. G. Yu, J. J. Xie, J. Yang, H. J. Huang, K. Wang and Z. S. Wen, J. Electroanal. Chem., 2004, 573, 121.

18 B. C. Duan, W. K. Wang, A. B. Wang, K. G. Yuan, Z. B. Yu, H. L. Zhao, J. Y. Qiu and Y. S. Yang, J. Mater. Chem., A 2013, 1, 13261.

19 K. Helios, A. Pietraszko, W. Zierkiewicz, H. Wojtowicz and D. $95 \quad$ Michalska, Polyhedron, 2011, 30, 2466.

20 K. Grenader, M. Kind, L. Silies, A. Peters, J. W. Bats and M. Bolte, J. Mol. Stru., 2013, 1039, 61.

21 X. W. Wang, Z, A, Zhang, Y. H. Qu, Y. Q. Lai and J. Li, J. Power Sources, 2014, 256, 361.

10022 J. Yang, J. Xie, X. Y, Zhou, Y. L. Zou, J. J. Tang, S. C. Wang, F. Chen and L. Y. Wang, J. Phys. Chem. C., 2014, 118, 1800. 